\title{
MICROTEXTURE, GRAIN BOUNDARY AND GRAIN JUNCTION EVOLUTION IN AI-Li SHEET DURING SUPERPLASTIC FORMING
}

\author{
VALERIE RANDLE and SIMON G. LEWIS \\ Dept. of Materials Engineering, University of Swansea, Swansea, SA2 8PP, UK
}

(Received 30 October 1995; in final form 19 December 1995)

\begin{abstract}
The microtexture of superplastically deformed $8090 \mathrm{Al}-\mathrm{Li}$ sheet has been measured using electron backscatter diffraction, for true strains of $0,0.25,0.75,1.5$ and 2.4. The data has been interpreted in terms of individual texture variants, grain boundary types (low angle or high angle) and grain junction types (I-lines or U-lines, as defined by an extension to the O-lattice theory). A key feature of the results is that local textures tend to be dominated by only one or perhaps two orientation variants, which leads directly to the presence of a high proportion of low or medium angle boundaries and hence a high proportion of I-line grain junction types. These effects decrease with increasing superplastic strain. Since it has been previously suggested that the presence of I-lines indicates dislocation-related phenomena, and the presence of U-line grain junction types denotes diffusion-related phenomena, the proportion of each of these provides as indication of the predominant accommodation mechanism.
\end{abstract}

KEY WORDS: Grain junctions, grain boundaries, microtexture, orientation variants, superplastic deformation.

\section{INTRODUCTION}

Previous work has indicated that the texture of Al-Li sheet changes considerably during superplastic deformation under biaxial loading (Bowen, 1988). In general, the rotation and sliding of grains by which superplastic flow takes place is manifested by weakening of the texture. However, it is also argued that where the deformation process is dominated more by slip or dislocation creep (Ashby and Verall, 1973) (i.e. at high stresses and/ or high strain rates), texture will be retained. Evidence from the microstructure and texture examination in the reference by Bowen (1988) suggests that slip occurs in the early stages of deformation, since some grains are elongated and so cannot rotate easily, and that this is accompanied by a small amount of texture strengthening. However, once grains became equiaxed they were free to rotate and slide, and a decrease in texture resulted. Hence texture changes are indicative of the processes which are occurring during superplastic flow.

Other work on Al-Li sheet which had undergone the same superplastic deformation as in the work by Bowen (1988) has focussed on local texture effects, in particular the characteristics of grain boundaries and grain junctions after true strains in excess of 2.4 (Randle, 1995a). It is well established that grain boundaries are the key microstructural elements in superplasticity because grain boundary sliding/rotation underpins the entire phenomenon (Raj, 1988). The importance of grain junctions in this context is that these are the sites at which relaxation processes known loosely as 
'accommodation' must take place in order to expedite grain rotation/sliding and thus sustain superplastic flow (Langdon, 1991). Grain junctions (or 'triple lines') have been studied less frequently than have grain boundaries, both in superplastic materials and as the subject of microstructural investigations in general.

Evidence from the interfacial (i.e. grain boundary and grain junction) cavitation which was reported in the work by Randle (1995a) showed that high angle boundaries and U-lines are more resistant to cavitation than are low angle boundaries (LABs) and Ilines. 'I-line' refers to the designation of a grain junction as low energy because a dislocation balance situation exists and conversely U-lines are higher energy, tube-like defects. The theory for the calculation is based on the O-lattice model (Bollmann, 1988, 1991) and the methodology for obtaining I-line/U-line classifications from orientation data has been described elsewhere (Palumbo and Aust, 1989; Randle, 1993a, 1994, 1995a). Furthermore, it has been proposed that the proportion of I-lines and U-lines present after plastic deformation was indicative of the dominant deformation mechanism: a high proportion of I-lines was associated with dislocation mechanisms (slip, dislocation creep) whereas U-lines were associated with diffusion accommodated flow. Although an I-line can result at the junction of three high angle boundaries, the probability of producing an I-line increases as the misorientation angle between the component boundaries at the junction decreases. It has been shown previously that the proportion of I-lines correlates with the proportion of 'small-medium angle boundaries' (SMABs) i.e. those having a misorientation $\leq 25^{\circ}$ (Randle, 1995b).

The first of the investigations mentioned above on texture changes during superplastic flow an $\mathrm{Al}-\mathrm{Li}$ sheet used X-ray techniques to obtain the texture data, giving a macroscopic view of the texture in the sheet for ascending levels of strain (Bowen, 1988). The second investigation outlined above (Randle, 1995a) used electron backscatter diffraction (EBSD) to measure the 'microtexture' (i.e. grain-by-grain texture) in a single specimen having been deformed $>2.4$ true strain, thus allowing microstructure-level information on orientation clustering and interface crystallography to be acquired and analysed. The present work extends the two investigations previously conducted by examining the microtexture of $\mathrm{Al}-\mathrm{Li}$ sheet having been deformed over a range of strains with the aim of following the microstructural evolution in terms of changes in the local orientation, grain boundary and grain junction populations.

\section{EXPERIMENTAL}

A sheet of $8090 \mathrm{Al}-\mathrm{Li}$ alloy was superplastically deformed under biaxial loading into the shape of a cone. Details of the alloy and forming conditions are given by Bowen (1988). Specimens were cut from the cone so as to represent true strains of $0.25,0.75$, 1.5 and 2.4, denoted specimens A, B, C and D respectively. In addition, one specimen was cut from the flange of the sheet material prior to superplastic deformation in order to supply 'control' data. This specimen was denoted $O$. All specimens were metallographically mounted with the original rolling plane parallel to the specimen surface, then polished to $1 / 4$ sheet thickness and etched in Keller's reagent before collecting orientations of individual grains using EBSD in a JEOL 6100 scanning electron microscope. Data were collected from contiguous grains in sampled regions of microstructure. In addition, sample populations of non-contiguous, dispersed grains were acquired from the superplastically deformed specimens (A-D) in order to mimic global, rather than local, sampling. 
In total the orientation of nearly 2000 grains was measured, yielding $\approx 3000$ grain misorientations and $\approx 2100$ grain junctions. Proportions of texture variants (using a $<20^{\circ}$ spread), grain misorientations and grain junction parameters were computed using in-house software.

\section{RESULTS}

Figure 1 shows 111 microtexture pole figures from each specimen. It can be seen that the starting texture (specimen $\mathrm{O}$ ) is weak, but is replaced by a very strong texture after 0.25 true strain (specimen A). Further deformation is characterised by a progressive weakening of the local textures.

The orientations were quantitatively analysed, revealing that the orientations present were

(1) S-texture, $\{123\}<412>$ or $\{123\}<634>$;

(2) Brass texture, $\{110\}<112>$;

(3) $\{102\}<221>$, which is a twin of the rotated cube texture $\{120\}<001>$, and so will be designated RT.

The S and RT textures comprise four variants each, S1, S2, S3, S4 and R1, R2, $\mathrm{R} 3$, R4 respectively whereas the brass texture comprises two variants, B1, B2. These ten variants are:

$\begin{array}{llllll}\text { S1 } & (123)[41 \overline{2}] & \text { R1 } & (102)[\overline{2} 21] & \text { B1 } & (110)[1 \overline{1} 2] \\ \text { S2 } & (123)[\overline{4} \overline{1} 2] & \text { R2 } & (102)[2 \overline{2} \overline{1}] & \text { B2 } & (110)[1 \overline{1} \overline{2}] \\ \text { S3 } & (213)[14 \overline{2}] & \text { R3 } & (102)[22 \overline{1}] & & \\ \text { S4 } & (213)[\overline{1} \overline{4} 2] & \text { R4 } & (102)[\overline{2} \overline{2} 1] & & \end{array}$

Figure 2 shows the orientation distribution for each superplastically deformed specimen using a $\pm 20^{\circ}$ tolerance. It can be seen that there are differences between the orientation distributions of each specimen on the basis of individual orientation components. For example, although the S-texture is the major texture type observed in each specimen, in specimens $\mathrm{A}$ and $\mathrm{C}$ the $\mathrm{S} 4$ variant predominates, whereas in specimens $B$ and $D$ a mixture of $S 1 / S 2$ and $S 1 / S 3$ respectively predominates. The overall trend is for a single texture variant in each of $S$, Brass and RT to dominate the sampled local microtexture. Also included in Figure 1 are 111 pole figures from the superplastically deformed specimens representing global, rather than local, orientation sampling.

The 'raw' orientation data were further processed to produce proportions of I-lines, LABs (misorientation $<15^{\circ}$ ) and SMABs (misorientation $<25^{\circ}$ ). Figure 3 shows these data plotted as a function of true strain. It is apparent that all three parameters decrease with increasing strain and there is a strong correlation between the proportion of I-lines and both LABs and SMABs. SMABs are included for comparison with computer simulated data (see next section).

Figure 4 shows an example of the microstructure for specimen $C$. The grain size distribution did not change substantially during the deformation. The surface pitting is a result of the overetching which was necessary both to obtain good diffraction patterns and furthermore to delineate every boundary. High angle boundaries often etched more deeply than LABs, and as a consequence of this etching effect strongly delineated elongated regions containing grains interfaced by LABs were observed in the 

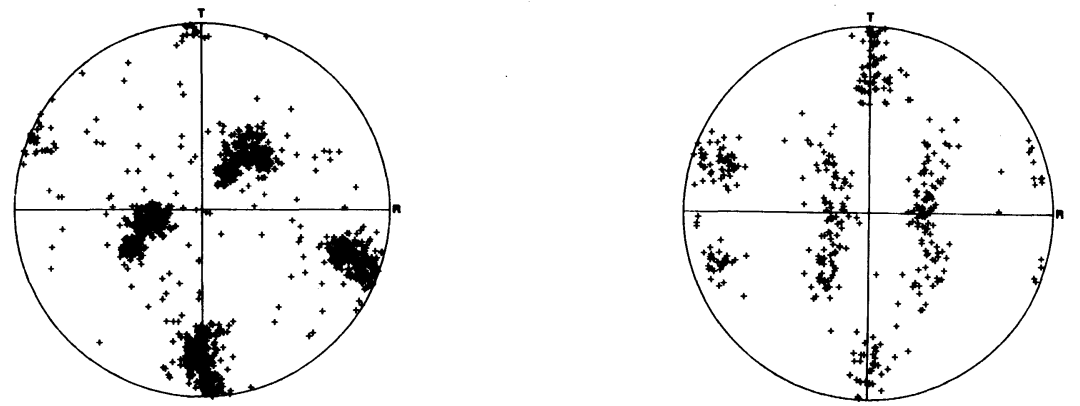

A

A
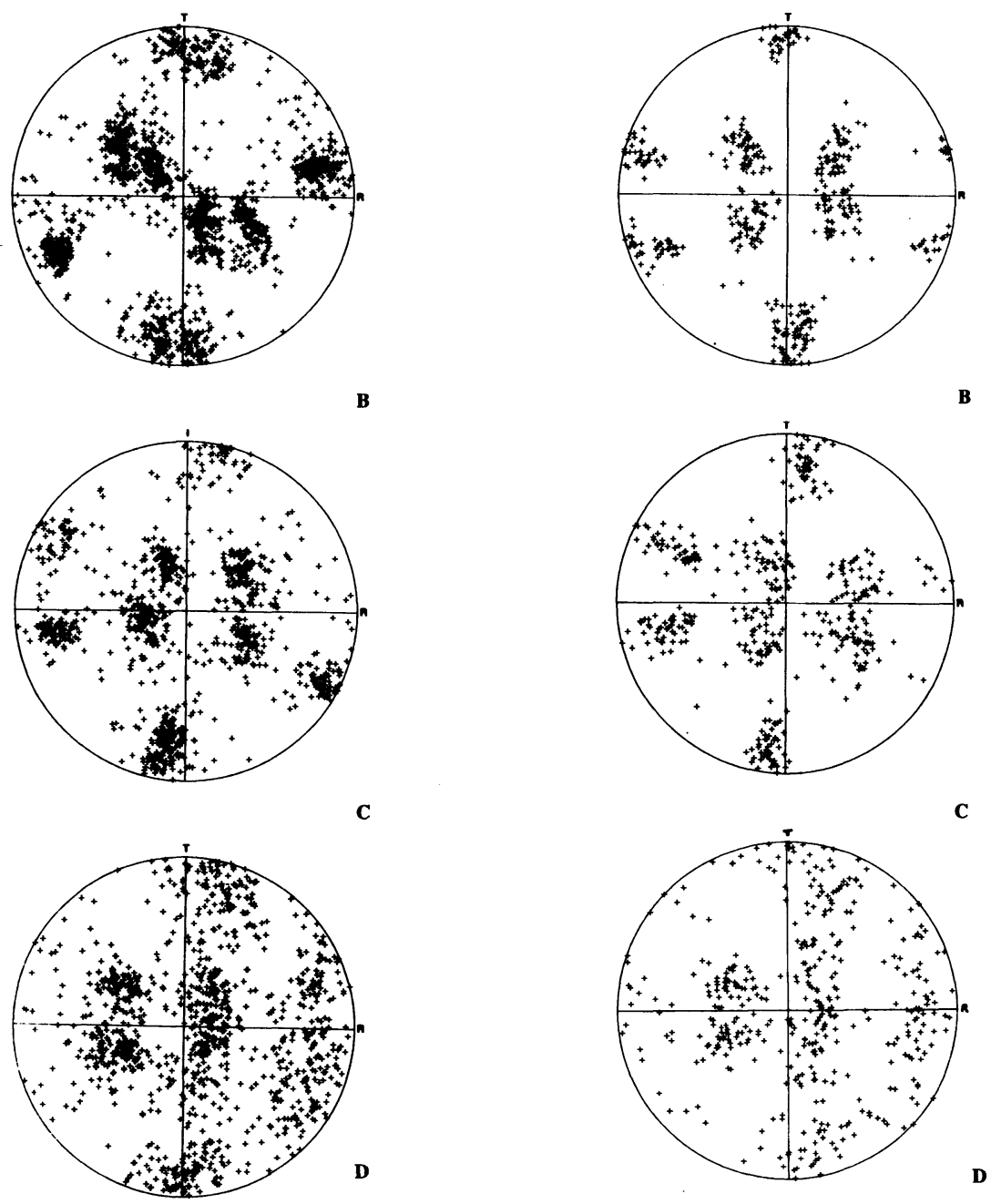

Figure 1a 111 microtexture pole figures for specimens A-D sampled on a local basis (left hand column), accompanied by 111 pole figures for these specimens sampled on a global (i.e. step sampling) basis (right hand column). 


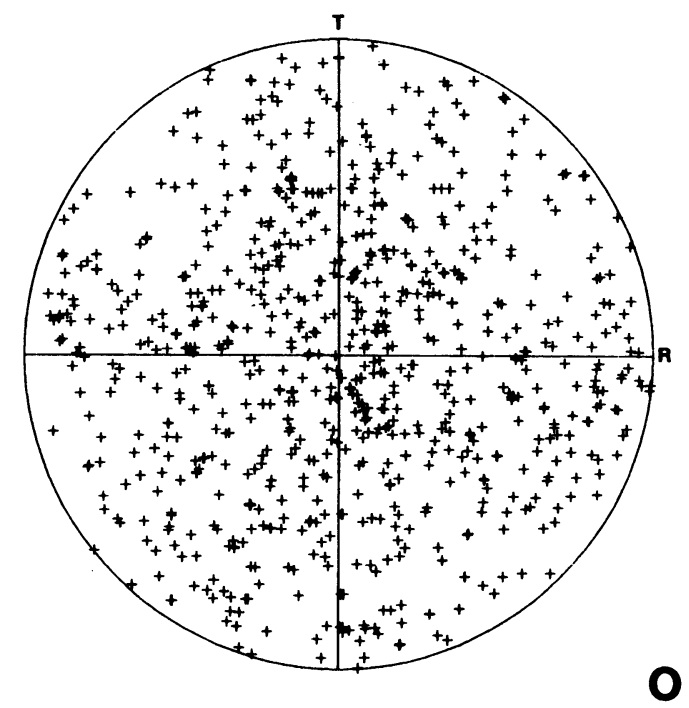

Figure 1b The 111 microtexture pole figure for the control specimen, specimen $\mathrm{O}$.

microstructure as shown in Figure 4. Usually the misorientation across these LABs was $5-15^{\circ}$, therefore they were not 'subboundaries'.

Figure 5 shows a microtexture 'map', that is, an area of which Figure 4 is a subset and which has been reproduced schematically in order to show the connectivity of texture component types, boundary types and I-lines. The texture variants are also shown, and it can be seen that specific texture variants often occur in clusters.

\section{DISCUSSION}

Some of the texture characteristics which were revealed by $\mathrm{X}$-ray measurements in (Bowen, 1988) as a function of strain, are also observed here, namely that there is an initial increase in texture at the onset of superplastic deformation (specimen $\mathrm{A}$ compared to specimen $\mathrm{O}$ ) followed by an overall decrease in texture as deformation proceeds. The RT texture was not observed using X-rays, and it is possible that this component was masked by the brass texture. Furthermore the texture decrease with strain is less marked for the present data than for the X-ray case, which may be because here a $20^{\circ}$ texture tolerance was chosen to categorise the data, and also the present measurements were acquired at $1 / 4$ thickness of the sheet rather than $1 / 2$ thickness as in the X-ray case.

A key feature of Figures 1 and 2 is the diversity in texture variants which was recorded. Such differences are not apparent in the X-ray data. When microtexture data is collected from a specific region in the microstructure it may not necessarily reflect the macrotexture, since local areas of orientation clustering or grouping are often present (Randle, 1993b). However, if EBSD is used to sample grains on a regular step basis rather than on a contiguous basis, we can obtain also an estimation of the global texture. 

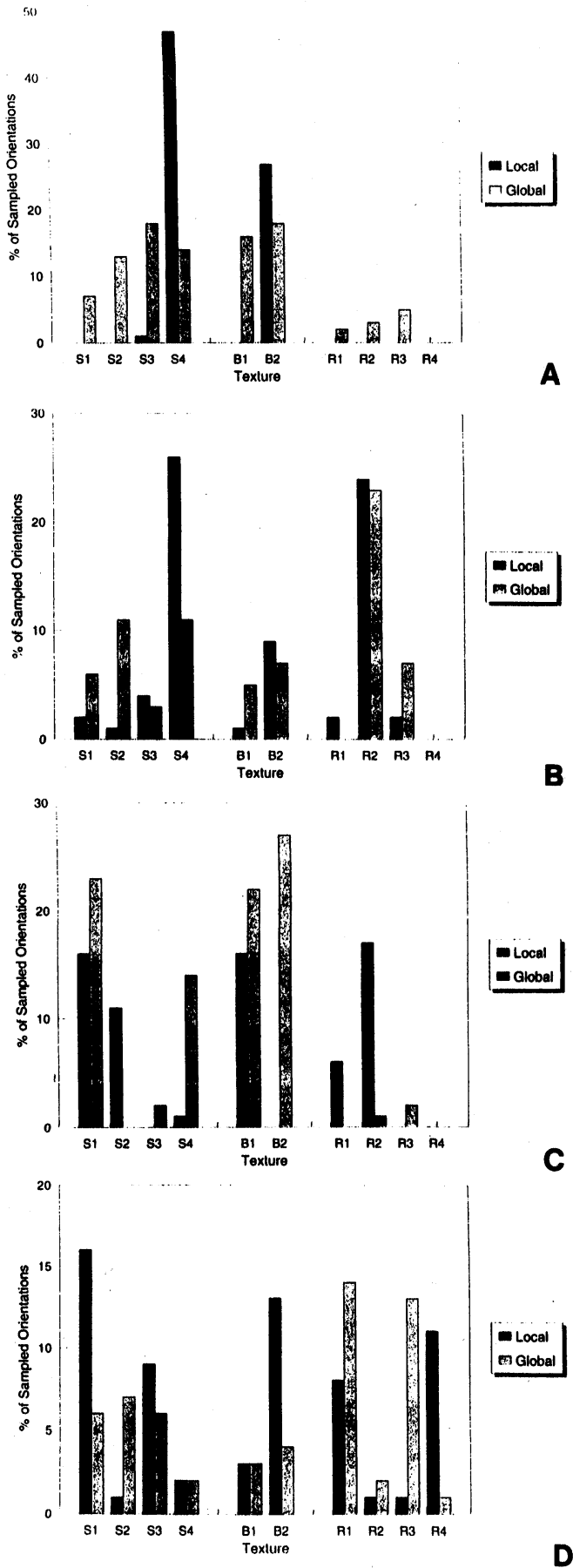

Figure 2 Proportions of texture components for specimens A-D for both local and global sampling. 


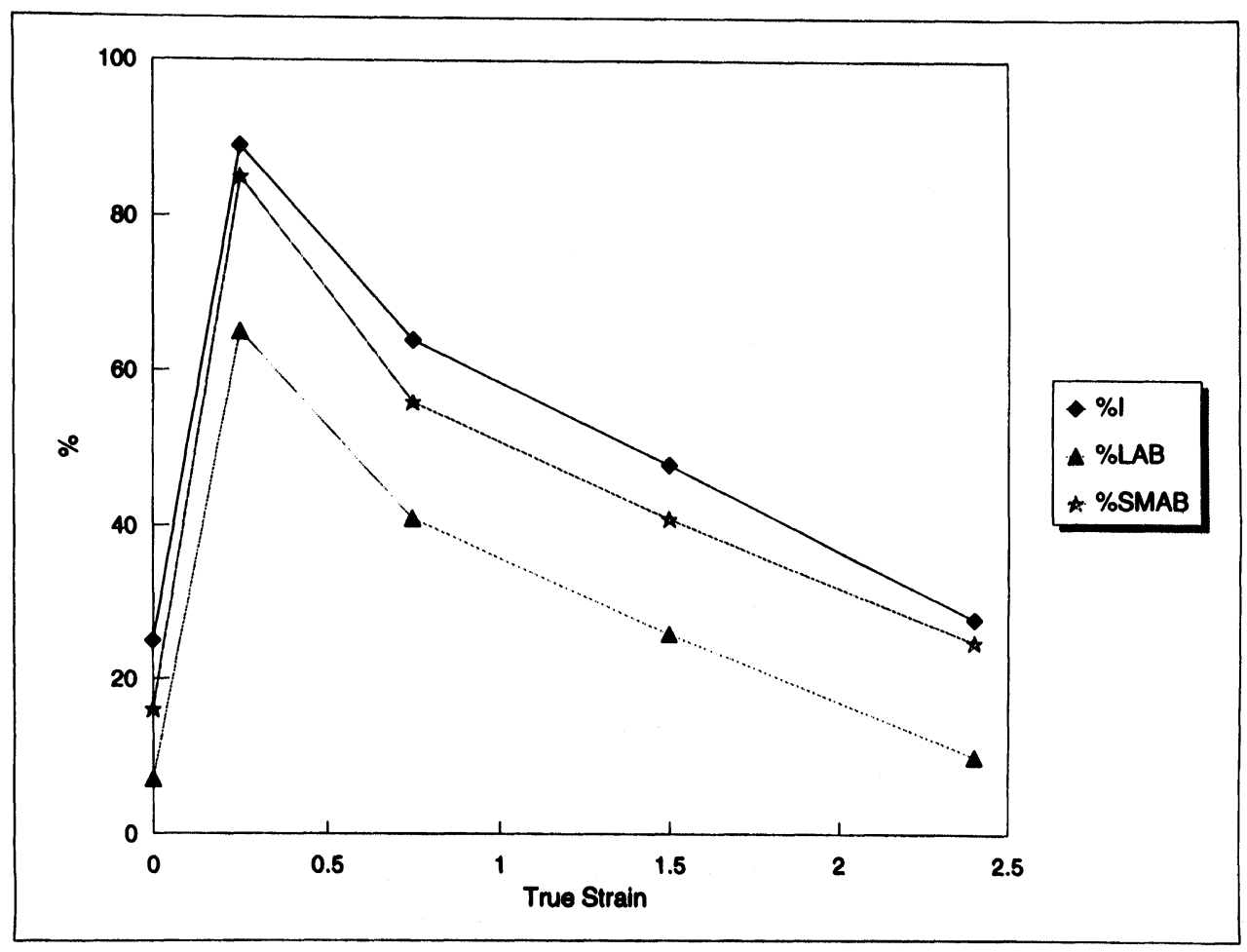

Figure 3 Variation of I-line, LAB and SMAB proportions with applied true strain.

For each superplastically deformed specimen a microtexture was obtained in this global manner by sampling orientations every $100 \mu \mathrm{m}$ in linear scans. The 111 pole figures for these data sets are included in Figures 1 and 2. It can be seen that the microtexture is spread more evenly between most of the texture variants in the globally sampled data sets than in those which were sampled locally. In other words, there is orientation clustering on a local scale in the material, particularly with respect to dominance of one or perhaps two particular texture variants.

Since formally the misorientation distribution is a re-expression of the parent orientations, the particular variant(s) of texture components which occurs will affect markedly the resulting misorientations. For example, two S1 orientation occurring as neighbouring grains will have a LAB between them whereas S1 and S2, or S3 and S4 orientations occurring as neighbours are related by a $60^{\circ}$ (twin) misorientation (Randle, 1993b), i.e. a $\Sigma=3$ boundary in coincidence site lattice (CSL) notation. Similarly B1 and B2 are twin-related. Moreover, small/medium angle boundaries result from the juxtaposition of several other variants, e.g. B1 and S1, B2 and S3, R1 and B1 are among those having a misorientation of about $20^{\circ}$. A study of Figure 5 , the microtexture 'map', shows that most LABs are the result of the same texture variants occurring as neighbours, although because a $20^{\circ}$ tolerance has been used to classify texture variants, sometimes they are not linked by a LAB. The largest single variant group in Figure 5 comprises 21 linked grains with S4 orientations. 


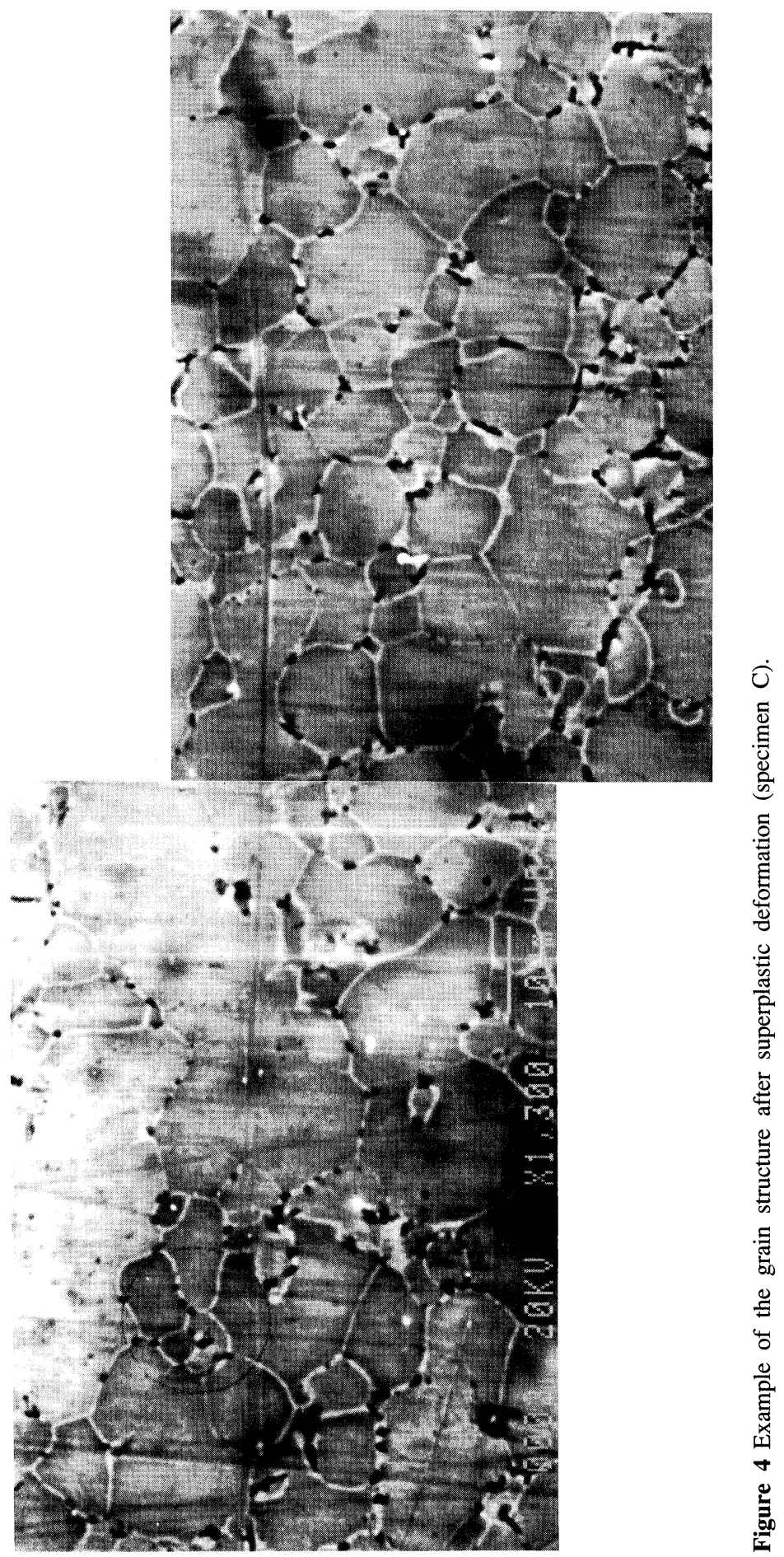




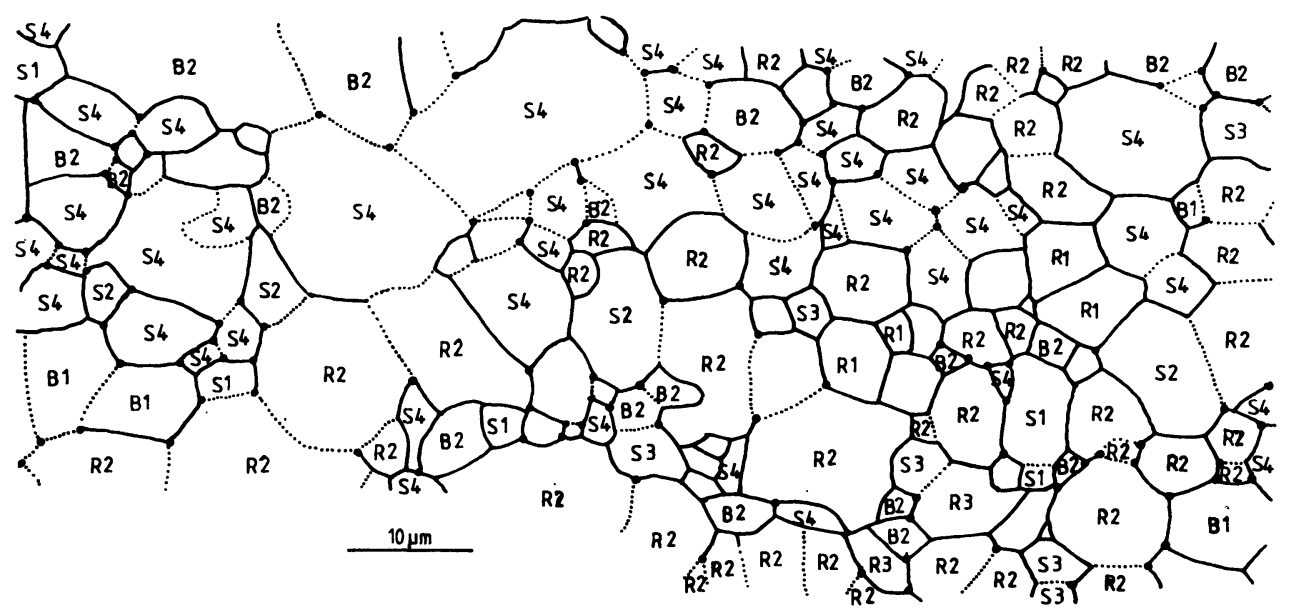

Figure 5 Microtexture 'map' (traced from Figure 4) showing the locations of LABs (dotted lines), high angle boundaries (full lines), I-lines (circles) and U-lines (no symbol). The texture variant of each grain is also labelled.

It is clear then that the misorientation angle distribution will be heavily biased towards small angles where the number of S, B and R texture variants is limited in a region. These effects are directly responsible for the distribution of LABs and SMABs in the data sets. For example, specimen B, which contains $85 \%$ SMABs, has three-quarters of its orientations represented by either S4 or B2. In addition to the fact that many of the grains will have like-textured neighbours, the misorientation between S4 and B2 is $20^{\circ}$. Hence a high proportion of SMABs is bound to occur in this data set. From Figure 3 we see that the trend for decreasing proportions of LABs and SMABs with increasing strain is very strong and is entirely a consequence of the texture.

There is a strong correlation between the proportion of I-lines and SMABs. Computer simulation of orientations in trios has shown that there is a sigmoidal relationship between the I-line and SMAB fraction, and over the linear part of the curve the ratio I/SMAB is 1.26 (Randle, 1995b). For the superplastically deformed specimens examined here this ratio ranges from 1.05 to 1.17 , which is in good agreement with the simulated data.

We turn now to the effect of the measured microstructural parameters on the mechanisms of superplastic deformation in Al-Li sheet. The high proportion of LABs in the early stages of deformation was not predicted from previous texture work, since $\mathrm{X}$-ray data does not supply the information necessary to compute the grain boundary parameters. In general LABs are resistant to sliding and migration, although previous work has shown that LABs with misorientations $5-15^{\circ}$ are capable of sliding during superplastic deformation (Matsuki, Iwaki, Tokizawa and Murakami, 1991). However, the rate of sliding for high angle boundaries and LABs is likely to be different, suggesting that grains divided by LABs deform as a group, with rotation in addition to the occurrence of sliding in order to maintain continuity at high angle boundaries.

The high proportion of I-lines present at low strains as a consequence of SMAB interactions suggests that slip can occur easily since dislocations can flow through an I-line exactly as if they were in a single crystal (Bollmann, 1988, 1991. It has been 
suggested that dislocation creep can occur also readily at I-lines for the same reason. It is therefore proposed that the mechanism for the continuation of superplastic flow at low strains is controlled by dislocation absorption and transmission at I-lines, which in turn is controlled by the special structure of I-lines which involves the balance of dislocations from the three component grain boundaries.

As superplastic deformation proceeds, the proportion of I-lines reduces with strain to $28 \%$ (Figure 3), and at this high strain the triple junction distribution is mainly dominated by U-lines. U-lines have an open structure and therefore are easy diffusion paths, like high angle boundaries, and so will facilitate the accommodation processes at grain junctions which must accompany grain rotations. Conversely, I-lines and LABs have lower diffusivities. The reduction in the proportion of I-lines and LABs as superplastic flow proceeds could indicate a progressive change in the major deformation mechanism from dislocation creep/slip to diffusion creep in the sampled regions.

\section{CONCLUSIONS}

1. Microtexture measurements from superplastically deformed $\mathrm{Al}-\mathrm{Li}$ sheet show that the texture varies on a local scale, and in the sampled regions only a few texture variants dominate thus giving rise to a high proportion of LABs.

2. In the early stages of superplastic deformation the grain interface distribution, i.e. grain boundaries and grain junctions, is dominated by I-lines and LABs. These proportions decrease with increasing strain.

3. It is suggested that as superplastic deformation proceeds the dominant mechanism changes from dislocation related phenomena (slip, dislocation creep), which is facilitated by a high proportion of I-lines, to diffusional processes which favour high angle boundaries and U-lines to effect accommodation at grain junctions.

\section{References}

Ashby, M. F. and Verrall, R. A. (1973). Diffusion Accommodated Flow and Superplasticity. Acta Met., 21, 149-159.

Bollmann, W. (1988). Triple-line disclinations. Representations, continuity and reactions. Phil. Mag., 57A, 637-649.

Bollmann, W. (1991). Triple-line disclinations in polycrystalline material. Mat. Sci. Eng., A136, 1-7.

Bowen, A. W (1988). Texture Changes in Al-Li Alloy Sheet superplastically deformed to high Strains. Tex. and Micros., 8 \& 9, 233-246.

Langdon, T.G. (1991). The role of grain boundaries in high temperature deformation. Mat. Sci. Eng., A127, 1-18.

Matsuki, K., Iwaki, T., Tokizawa, M. and Murakami, Y. (1991). Microstructural evolution during initial stage of high strain rate superplastic deformation in powder metallurgical $7475 \mathrm{Al}-0.7 \mathrm{Zr}$ alloy. Mat. Sci. Tech., 7, 513-519.

Raj. R. (1988). Relationships between interface structure and superplastic deformation. J. de Phys., 49, C5-35-47.

Randle, V. (1993a). On the significance of grain junction topology and crystallography in polycrystals. Scripta Met. Mat., 28, 889-893.

Randle, V. (1993b). A microtexture investigation of cracking in Al-Li sheet. Tex. and Micros., 21, 219-232.

Randle, V. (1994). Grain assemblage in polycrystals (overview). Acta Met. Mat., 42, 1769-1784.

Randle, V. (1995a). The influence of grain junctions and boundaries on superplastic deformation. Acta Met. Mat., 43, 1741-1749.

Randle, V. (1995b). Acta Met. Mat., in press. 